\title{
Síndrome de sobreposição lúpus eritematoso sistêmico e esclerodermia: relato de caso e revisão de literatura
}

\author{
Systemic erythematosus lupus overdose syndrome and sclerodermia: case report and \\ literature review
}

Síndrome de sobreposición lupus eritematoso sistémico y esclerodermia: relato de caso y revisión de literatura

Carla Daniele Nascimento Pontes ${ }^{1 *}$, Yuri José Almeida Da Silva ${ }^{2}$, Rodrigo Bona Maneschy ${ }^{1}$, Lucianna Serfaty de Holanda ${ }^{1}$, Valeria da Silva Campos ${ }^{1}$, Karen Giovana Leal Matos ${ }^{1}$, Natalia Rodrigues Eugenio ${ }^{1}$, Natalia Xavier Silva Chini ${ }^{1}$, Brenna Pinheiro Mota Brabo de Oliveira ${ }^{1}$, Íris Malato Corrêa ${ }^{1}$, Ivy de Almeida Cavalcante e Silva ${ }^{1}$, Carolina Tavares Carvalho', Ian Silva Rodrigues ${ }^{1}$, Faisal Mesquita Salmen Hussain ${ }^{1}$, Rudival Faial de Moraes Junior ${ }^{1}$.

\section{RESUMO}

Introdução: O lúpus eritematoso sistêmico (LES) é uma doença inflamatória crônica, multissistêmica, de causa desconhecida e de natureza auto-imune e evolui com manifestações clínicas polimórficas, com períodos de exacerbações e remissões. A Esclerose Sistêmica (ES) é uma doença autoimune, de etiologia desconhecida, que afeta o tecido conjuntivo. A concomitância de síndromes que afetam o tecido conjuntivo já é conhecida, uma vez que as manifestações nem sempre são restritas as características de uma única doença. Detalhamento do caso: Paciente de 47 anos já diagnosticada com Lúpus Eritematoso Sistêmico estava em acompanhamento regular quando iniciou quadro de dispneia progressiva e disfagia. Foi internada para investigação clínica e observou-se em exame radiológico de imagem presença de fibrose pulmonar, além de esofagopatia. A paciente já possuía episódios frequentes de fenômeno de Reynoud e esclerodactilia. Com a evolução do quadro em associação com as alterações que a paciente já possuía foi fechado o diagnóstico de Esclerose Sistêmica em sobreposição ao quadro de Lúpus eritematoso sistêmico. Discussão: O Lúpus Eritematoso Sistêmico (LES) é uma complexa doença autoimune com um largo espectro clínico e imunológico. A ES pode acometer vários órgãos e sistemas, sendo a pele o órgão mais afetado, seguida dos pulmões, sistema musculoesquelético, rins, coração e trato gastrointestinal. A sobreposição dessas patologias configura um caso raro que demanda identificação rápida do profissional de saúde a fim de minimizar dados e complicações de ambas as patologias.

Palavras-chave: Lúpus Eritematoso Sistêmico, Esclerodermia, Overlap.

\section{SUMMARY}

Introduction: Systemic lupus erythematosus (SLE) is a chronic, multisystemic inflammatory disease of unknown cause and autoimmune in nature and evolves with polymorphic clinical manifestations, with periods of exacerbations and remissions. Systemic sclerosis (ES) is an autoimmune disease of unknown etiology that affects connective tissue. The concomitance of syndromes that affect the connective tissue is already known, since the manifestations are not always restricted to the characteristics of a single disease. Case report: A 47-year-old patient diagnosed with Systemic Lupus Erythematosus was regularly followed up when she started progressive dyspnea and dysphagia. She was admitted for clinical investigation and the presence of pulmonary

${ }^{1}$ Fundação Santa Casa de Misericórdia do Pará, Belém-PA. * E-mail: pontes4@live.com

2 Universidade Federal do Pará, Belém-PA. 
fibrosis, in addition to esophagopathy, was observed on radiological imaging. The patient already had frequent episodes of Reynoud's phenomenon and sclerodactyly. With the evolution of the condition in association with the changes that the patient already had, the diagnosis of Systemic Sclerosis was closed in overlapping with the systemic lupus erythematosus. Discussion: Systemic lupus erythematosus (SLE) is a complex autoimmune disease with a broad clinical and immunological spectrum. ES can affect several organs and systems, the skin being the organ most affected, followed by the lungs, musculoskeletal system, kidneys, heart and gastrointestinal tract. The overlapping of these pathologies is a rare case that demands the rapid identification of the health professional in order to minimize data and complications of both pathologies.

Key words: Systemic Lupus Erythematosus, Scleroderma, Overlap.

\section{RESUMEN}

Introducción: El lupus eritematoso sistémico (LES) es una enfermedad inflamatoria crónica, multisistémica, de causa desconocida y de naturaleza autoinmune y evoluciona con manifestaciones clínicas polimórficas, con períodos de exacerbaciones y remisiones. La Esclerosis Sistémica (ES) es una enfermedad autoinmune, de etiología desconocida, que afecta el tejido conectivo. La concomitancia de síndromes que afectan al tejido conjuntivo ya es conocida, ya que las manifestaciones no siempre son restringidas a las características de una sola enfermedad. Detalle del caso: Paciente de 47 años ya diagnosticada con Lupus Eritematoso Sistémico estaba en seguimiento regular cuando inició cuadro de disnea progresiva y disfagia. Fue internada para investigación clínica y se observó en un examen radiológico de imagen presencia de fibrosis pulmonar, además de esofagopatía. La paciente ya poseía episodios frecuentes de fenómeno de Reynoud y esclerodactilia. Con la evolución del cuadro en asociación con las alteraciones que la paciente ya poseía fue cerrado el diagnóstico de Esclerosis Sistémica en superposición al cuadro de Lupus eritematoso sistémico. Discusión: El Lupus Eritematoso Sistémico (LES) es una compleja enfermedad autoinmune con un amplio espectro clínico e inmunológico. La ES puede acometer varios órganos y sistemas, siendo la piel el órgano más afectado, seguido de los pulmones, sistema musculoesquelético, riñones, corazón y tracto gastrointestinal. La superposición de estas patologías configura un caso raro que demanda identificación rápida del profesional de salud a fin de minimizar datos y complicaciones de ambas patologías.

Palabras clave: Lupus Eritematoso Sistémico, Esclerodermia, Overlap.

\section{INTRODUÇÃO}

O lúpus eritematoso sistêmico (LES) é uma doença inflamatória crônica, multissistêmica, de causa desconhecida e de natureza auto-imune, caracterizada pela presença de diversos auto-anticorpos. Evolui com manifestações clínicas polimórficas, com períodos de exacerbações e remissões. O desenvolvimento da doença está ligado a predisposição genética e fatores ambientais, como luz ultravioleta e alguns medicamentos (BORBA et al, 2008). Esta doença possui períodos de exacerbação e remissão, tornando mais difícil o manejo médico (RUS, 2007). Os custos estimados para o tratamento da doença são elevados e estão diretamente relacionados à gravidade desta, devido ao tipo de medicamento diferenciado e o número de exacerbações dos casos mais severos. (DORIA et al, 2015)

Dentre as formas de apresentação da doença, podemos diferenciar o Lúpus Eritematoso Sistêmico e o Lúpus Eritematoso Cutâneo (LEC), apesar destas duas formas não serem sempre tão bem diferenciadas na literatura científica, haja vista que existem várias intercessões entre ambas. Apesar das lesões de pele específicas das 4 subclassificações do LEC, existem manifestações comuns compartilhadas com a LES, como Fenômeno de Raynaud, telangiectasiasperiungueais e vasculites (KUHN, 2006).

Segundo estudos norte-americanos, a doença afeta mais frequentemente mulheres jovens (9-10:1) com sua prevalência variando de 14 a 50/100.000 habitantes (RUS, 2007). Estudos envolvendo a população 
brasileira observou incidência mais elevada em pacientes brancos e também em mulheres jovens (ADINOLFI et al, 2016).

A esclerodermia é uma doença autoimune, cujas manifestações clínicas podem ser bastante variáveis. Algumas formas são mais brandas, enquanto outras podem ser letais. A esclerodermia pode ser classificada de duas maneiras: Esclerose Sistêmica - que causa fibrose, com acometimento cutâneo, vascular e de órgãos internos; é mais comum em adultos - e Esclerodermia localizada - que causa fibrose na pele e tecidos adjacentes, sem acometimento vascular ou de órgãos internos; é mais comum em crianças (TOROK, 2012).

A Esclerose Sistêmica (ES) é uma doença autoimune, de etiologia desconhecida, que afeta o tecido conjuntivo. Trata-se de uma condição rara, potencialmente fatal, cuja incidência, segundo estudos realizados na Inglaterra, tem uma estimativa anual de 0,6 a 19 casos novos para cada milhão de habitantes. Em relação à prevalência, estudos realizados nos Estados Unidos e na Inglaterra apontam, aproximadamente, 1 paciente para cada 1000 habitantes. No Brasil, há poucos estudos publicados sobre a incidência e a prevalência de ES. Em um estudo realizado no Mato Grosso do Sul, em 2014, a taxa de incidência de ES foi de 11,9 casos por milhão de habitantes e a prevalência foi de 105,6 por milhão de habitantes (HORIMOTO et al., 2017).

$\mathrm{Na}$ ES, pode haver alterações caracterizadas por inflamação, fibrose e atrofia, associadas à endarterite proliferativa e lesões obstrutivas dos capilares, afetando pele, sistema músculo-esquelético e órgãos internos - como coração, pulmões e rins. As manifestações clínicas da ES são extremamente heterogêneas e o curso da doença pode ser bastante variável (KAYSER; ANDRADE, 2002).

É uma enfermidade que apresenta três subtipos clínicos, os quais estão relacionados a diferentes morbidades. O primeiro subtipo é o cutâneo difuso, rapidamente progressivo e caracterizado pelo espessamento generalizado da pele e pelo acometimento precoce de órgãos viscerais (coração, pulmões e rins). O segundo subtipo é o cutâneo limitado, caracterizado pelo espessamento restrito e não progressivo da pele, além de acometimento mais tardio de outros órgãos, causando, por exemplo, o desenvolvimento de hipertensão arterial pulmonar. O terceiro subtipo é a sobreposição de uma das formas clínicas (podendo ser cutâneo difuso ou cutâneo limitado) com outra doença que afete o tecido conjuntivo, a exemplo do Lúpus Eritematoso Sistêmico (LES) (TOROK, 2012).

A concomitância de síndromes que afetam o tecido conjuntivo já é conhecida, uma vez que as manifestações nem sempre são restritas as características de uma única doença. Desta forma, um paciente pode manifestar comemorativos não característicos a sua entidade patológica diagnosticada, mesmo que não preencha os critérios diagnósticos de uma outra; ou a variação seja tamanha que preencha, caracterizando a sobreposição (LIN, 2012).

Em estudo, observou-se que aproximadamente $30 \%$ dos pacientes apresentavam concomitância de LES com pelo menos mais uma outra doença autoimune, dentre elas, a esclerodermia (MCDONAGH, 2000).

Dito isto, este trabalho tem por objetivo relato de caso de uma paciente hospitalizado com diagnóstico de Síndrome de Sobreposição Lúpus Eritematoso Sistêmico e Esclerodermia.

\section{DETALHAMENTO DO CASO}

No ano de 2017, na Fundação Santa Casa de Misericórdia do Pará (FSCMPA), ocorreu um caso de sobreposição do Lúpus eritematoso sistêmico e Esclerodermia.

Em junho do ano supracitado, deu entrada no hospital paciente do sexo feminino, 47 anos, com diagnóstico de Lúpus há 8 anos, procedente do ambulatório de reumatologia onde fazia acompanhamento desde o diagnóstico da doença. Na admissão relatava dispneia em repouso, com piora aos pequenos esforços, cianose de extremidades, dor generalizada, principalmente articular, dor torácica ventilatório dependente, astenia, anorexia e referia 1 episódio de febre. Na manhã da internação, durante pulso com Metilprednisolona, evoluiu com piora dos sintomas e elevação dos níveis pressóricos, sendo encaminhada para internação. De antecedentes negava Hipertensão arterial e diabetes. Ao exame encontrava-se com cianose de extremidades, 
com Pressão arterial de 160×100 MMHG, com murmúrio vesicular discretamente diminuído em bases pulmonares. Foi diagnosticada com Lúpus eritematosos sistêmico, miositelúpica e Fibrose pulmonar, em uso de Prednisona $60 \mathrm{mg} / \mathrm{dia}$ que foi mantida.

Na internação evoluiu com fraqueza muscular proximal, refluxo gastroesofágico, com náuseas e vômitos persistentes, fenômeno de Raynaud, algia em membros inferiores. Fez uso prednisona $60 \mathrm{mg} / \mathrm{dia}$, contudo mantinha dispneia, fraqueza muscular proximal com dificuldade para deambular e dispneia, refluxo gastresofágico e fenômeno de Raynaud. Realizou pulsoterapiacom metilprednisolona e pulsoterapia com ciclosfofamida.

No decorrer da internação evolui com Pneumonia Nosocomial, mantinha a Fibrose pulmonar em bases com queixa de tosse produtiva com secreção purulenta, tomografia computadorizada de tórax apresentava espessamento de septos interlobulares difusos, associados a bandas parenquimatosas e bronquioloectasias de tração. Foi iniciada antibioticoterapia com Ceftriaxone e Claritromicina, sem melhora, e então tratada posteriormente com Piperacilina com Tazobactam sódico, obtendo resposta satisfatória após tratamento.

Devido a queixa de Refluxo Gastroesofágico, foi realizada Endoscopia digestiva alta, com resultado de esofagite edematosa moderada; monilíaseesofagiana severa, pangastrite endoscópica, tipo enantemática, edematosa, exsudativa de moderada intensidade, bulbodeniteenántemática, edematosa moderada. Tratada com Fluconazol por longo período.

Laboratório com sorologia para HIV negativas, FAN Padrão nuclear Pontilhado fino, Fator Reumatoide $<1$, Complemento C3: 90,26, Complemento C4: 16,52, ANTI-SCL: não reagente, ANTI JO: não reagente, Aldolase: 6,2; CPK, para diagnóstico de lesões e doenças da musculatura esquelética e também infarto agudo do miocárdio: 370 (aumentado), CPK 6 dias após o primeiro: 197 (limite superior de normalidadae) e Baar: negativo

Devido ao quadro de dor foi realizada Ressonância nuclear magnética de Coluna Lombo-sacra, na qual evidenciou um abaulamento discal posterior difuso nos níveis de L3-L4 e L4-L5. Tocando a face Ventral do Saco dural e reduzindo a amplitude de recessos neuroforaminais. Associa-se a rotura do ânulo fibroso de nível L4-L5. Injúria dos ligamentos interespinhosos nos níveis L3-L4 e L4-L5. Artrose interpofisária incipiente nos níveis de L3-L4 e L4-L5.

Foi realizado também um Ecocardiograma, na qual mostrou um aumento discreto das câmaras esquerdas, VE com desempenho global normal, Fração de ejeção de ventrículo esquerdo de 65\% (preservada), discreta regurgitação mitral e tricúspide

A paciente mantinha aumento de astenia, dor em grandes e pequenas articulações, parestesias, alopecia, dispneia aos esforços. E evoluiu com sopro sistólico em todos os focos e fácies típica de Esclerodermia e picos hipertensivos. Então realizada nova pulsoterapia com Ciclofosfamida e tratamento com Hidroxicloroquina.

Após mais de 2 meses de internação, com a terapia instituída, antibioticoterapia, fluconazol, prednisona, pulso com metilprednisolona, Hidroxicloroquina, recebeu alta com melhora, sem febre, dor ou dispneia e sem outras queixas, com diagnóstico de Síndrome de sobreposição Lúpus eritematoso sistêmico e Esclerodermia, devido a todo quadro apresentado.

\section{DISCUSSÃO}

O Lúpus Eritematoso Sistêmico (LES) é uma complexa doença autoimune com um largo espectro clínico e imunológico. É caracterizada pelo desenvolvimento de autoanticorpos e pelo autoataque imunológico a diversos sistemas. Tem etiologia desconhecida, mas está associada a fatores genéticos, hormonais e ambientais (MOREIRA, 2017).

Como fatores de risco, temos como exemplo o gênero feminino (tanto em lúpus cutâneo -LEC- quanto em lupus sistêmico-LES-); para fatores ambientais, não existe uma boa relação causal com o LES, mas há 
com a LEC (exposição aos raios UV, hábito de fumar); a variável étnica e socioeconômica influenciam na severidade e na mortalidade de ambas as formas patológicas (PONS-ESTEL et al, 2010); mutações genéticas como o gene TREX1, a deficiência de fatores do complemento (C1q e C4) e hiperexpressão de TYK2 podem também ser o caminho para uma melhor explicação sobre a etiologia desta doença (GÜNTHER et al, 2009; JÄRVINEN et al, 2012). A paciente do caso em questão era do sexo feminino e possui diagnostico de lúpus há 8 anos e encontra-se, portanto, dentro da faixa etária mais acometida pela doença.

Sobre a fisiopatologia, existem evidências de que a lesão difusa do lúpus seja de origem inflamatória vascular, haja vista que a patogênese da doença transpasse fundamentalmente por uma desregulação imunológica, causando a hiperproliferação de variados anticorpos, e a excitação das células endoteliais vasculares. Trata-se de um processo vicioso, no qual as células endoteliais ativadas liberam substâncias que coordenam uma resposta imunológica, da cascata de coagulação e do tônus vascular, os quais tem como resultado a ação inflamatória sobre o próprio endotélio, excitando-o novamente (BELMONT, 1996).

As manifestações clínicas da doença são extremamente variáveis, tanto no que tange a sintomatologia quanto em relação a expressão dos marcadores sorológicos da autoimunidade do paciente (ADINOLFI et al, 2016). Além disso, os danos irreversíveis, que leve a perda funcional já são esperados na evolução desta doença. (MARTINEZ-BARRIO et al, 2014).

Determinados órgãos podem ser costumeiramente atingidos pela LES (ADINOLFI et al, 2016). Segundo Bortoluzzi et al, 2014 dentre estas lesões, nós podemos salientar o espectro de síndromes neuropsiquiátricas. $\mathrm{Na}$ paciente do caso clínico de sobreposição, este acometimento não foi relatado durante o período de internação.

Segundo Mavragni et al 2015, outro acometimento descrito é a nefrite lúpica, na qual observou-se que casos mais severos de LES resultavam em nefrites do tipo III ou IV e casos mais brandos em nefrite tipo V. Na paciente do caso clinico não foram encontrados dados relacionando acometimento renal importante. Muito embora essa seja uma causa bastante comum de internação

Segundo Rothfiled 1995 e Belmont 1996, destacam-se dentro do escopo de sintomas respiratórios relacionados ao Lúpus Eritematoso Sistêmico, o envolvimento pleural (derrame pleural, pleurite), envolvimento vascular (hipertensão pulmonar, hemorragia alveolar), pneumonia intersticial e até lesões neurológicas como paralisia diafragmática. No caso clinico da paciente, observa-se que o acometimento respiratório que corrobora com a descrição do autor. Tendo em vista a descrição de sintomas como dispnéia, dor ventilatório dependente e alterações encontradas em exames de imagem relatados anteriormente, não relacionados somente a pneumonia que acabou acometendo a paciente.

O tratamento para LES é medicamentoso e tem se demonstrado com eficácia progressiva ao longo dos anos devido a novas estratégias terapêuticas associadas aos tratamentos tradicionais (ADINOLFI et al, 2016). O tratamento tradicional utiliza drogas antimaláricas (Hidroxicloroquina), com grande eficácia e grande utilização mundialmente (NORGAARD et al, 2015) e com drogas imunossupressoras (Ciclofosfamida ou Micofenolato) principalmente em pacientes com lesão renal (FASSBINDER et al, 2015; ADINOLFI et al, 2016). Existem ainda as drogas bloqueadores de células-B, como o Rituximab e o Belimumab. O Rituximab mostra uma redução na sua eficácia, provavelmente por mecanismo de resistência (REDDY et al, 2015); já o Belimumab demonstra uma grande vantagem quando utilizado em LES de instalação na faixa etária infantil (HUI-YUEN et al, 2015).

Dentre as formas de apresentação da doença, podemos diferenciar o Lúpus Eritematoso Sistêmico e o Lúpus Eritematoso Cutâneo (LEC), apesar destas duas formas não serem sempre tão bem diferenciadas na literatura científica, haja vista que existem várias intercessões entre ambas. Apesar das lesões de pele específicas das 4 subclassificações do LEC, existem manifestações comuns compartilhadas com a LES, como Fenômeno de Raynaud, telangiectasias periungueais e vasculites (KUHN, 2006). Estas descrições do estudo de Kuhn 2006 têm relevância dentro do caso relatado, pois a paciente já apresentava o fenômeno de Raynaud observado desde sua admissão. Embora este mesmo achado possa ocorrer também relacionada ao diagnóstico de esclerodermia que a paciente possuía. 
A Esclerose sistêmica (ES) é uma doença autoimune, de etiologia desconhecida e potencialmente fatal. Caracteriza-se por inflamação, disfunção vascular e fibrose da pele e de órgãos internos, devido à deposição excessiva de colágeno nos tecidos. É uma doença rara nos extremos de idade, sendo o pico de incidência durante a quinta década de vida. A sobrevida estimada para portadores dessa doença é de $66 \%$ em 10 anos. As duas principais causas de mortalidade relacionadas à essa patologia são insuficiência renal, em função da crise renal esclerodérmica, e a insuficiência pulmonar, devido à hipertensão pulmonar (ABRÃO et al, 2016). Esta paciente embora tenha diagnostico de duas patologias com risco de disfunção renal, não teve este acometimento como marco importante nesta internação

A ES pode acometer vários órgãos e sistemas, sendo a pele o órgão mais afetado, seguida dos pulmões, sistema musculoesquelético, rins, coração e trato gastrointestinal. $\mathrm{O}$ acometimento do sistema respiratório ocorre em 70 a $90 \%$ dos pacientes portadores, sendo as manifestações mais frequentes a doença pulmonar intersticial e a hipertensão arterial pulmonar (OLIVEIRA, 2017). A paciente apresentou durante a internação acometimento respiratório importante, com a constatação de fibrose pulmonar. Quanto ao acometimento cardiológico, a paciente do caso possui uma fração de ejeção de Ventrículo Esquerdo preservada, como constatado em ecocardiograma.

A ES é uma doença classificada em duas categorias, de acordo com a extensão de pele acometida. Há a ES limitada, na qual ocorre um espessamento da pele em áreas exclusivamente distais aos cotovelos e joelhos, podendo haver ou não acometimento da face. A "síndrome CREST" (caracterizada por calcinose, fenômeno de Raynaud, dismotilidade esofágica, esclerodactilia e telangiectasias) é um termo em desuso e atualmente enquadra-se na definição de ES limitada. Há, ainda, a ES difusa, na qual ocorre um espessamento da pele tanto a nível proximal como distal aos cotovelos e joelhos, podendo haver ou não envolvimento da face e do tronco (CHARLES; CLEMENTS; FURST, 2006).

Assim, o sinal clínico mais importante para diagnosticar ES é o espessamento da pele, que geralmente inicia com um edema da pele, normalmente nos dedos e nas mãos. O curso clínico da doença é bastante variável, sabendo-se que a forma cutânea difusa da doença é tipicamente mais agressiva (STEEN, 2001). A patogenia da doença, embora não esteja totalmente esclarecida, possui relação com uma complexa interação entre alterações vasculares com dano das células do endotélio, ativação da imunidade humoral e celular e disfunção no controle da síntese de tecido conjuntivo (PEREIRA et al., 2012; TOPAL; DHURAT, 2013).

A patogênese da doença está relacionada ao sistema vascular, às células endoteliais e adventícias; ao sistema conjuntivo, com os fibroblastos como célula central; e ao sistema imunológico, com fenômenos inflamatórios e autoimunes (STICHERLING, 2012). Como se trata de uma doença multissistêmica, que afeta de diversas maneiras e a níveis variados os diferentes órgãos e sistemas, não existem dois pacientes com manifestações idênticas, tornando extremamente importante a compreensão da sua patogênese, a fim de instituir a terapêutica mais adequada (TOPAL; DHURAT, 2013).

Segundo Lorenzoni e Martins 2010 estudos mostram que há uma predisposição genética a ser considerada para o desenvolvimento da doença. Embora tenha sido identificada uma distribuição familiar, não há evidências de padrão de herança mendeliano. Membros da mesma família apresentam uma incidência de $1,6 \%$ de manifestar a doença, em relação à população em geral. No caso da paciente, não foi encontrado dados que descrevessem acometimento de esclerodermia em familiares da mesma.

Suspeita-se que infecções, como citomegalovírus e parvovírus têm importância na etiopatogenia da ES. Suspeita-se também de substâncias utilizadas em certos pesticidas, de exposição a derivados de benzeno e àsílica, o que pode ser relevante na medicina ocupacional. (CHARLES; CLEMENTS; FURST, 2006) Na história da paciente não tiveram registros de acometimento por parte destas infecções ou exposição ocupacional que tem relação com a esclerodermia.

Acredita-se que um dos fatores de maior importância para o desenvolvimento da ES seja lesão endotelial que leva à disfunção vascular (HUMMERS et al., 2009). Mecanismos celulares que levam ao acúmulo de miofibroblastos na camada íntima dos vasos sanguíneos têm como repercussão o espessamento da camada e a consequente redução do lúmen vascular (CHARLES; CLEMENTS; FURST, 2006). Essa lesão endotelial 
pode ser agravada por mecanismos de isquemia e de reperfusão, resultando no aumento de diversas citocinas, tais como a endotelina-1 ou a prostaciclina. A ativação de diversas outras citocinas e receptores pode culminar no desequilíbrio vascular, causando vasoconstrição e fibrose (BALBIR-GURMAN; MOSCOVICl, 2012).

Segundo Kayser e Andredade 2002, o fenômeno de Raynaud é normalmente o sinal mais precoce da doença. Com a evolução da doença, surgem complicações devido ao envolvimento vascular de outros órgãos e os principais sintomas são fadiga e queixas musculo-esqueléticas, sobretudo artralgia ou artrite. $O$ diagnóstico ainda é obscuro nessa fase. O que sugere, mais especificamente, evolução para esclerose sistêmica é a presença de anticorpos antinúcleo (ANAs) ou de microangiopatia SD; idade tardia de início do fenômeno de Raynaud e grande intensidade do fenômeno. No caso relatado observa-se a ocorrência deste fenômeno, que também acontece no LES já diagnosticado.

A expressão fenotípica da doença é a fibrose. A fibrose da pele, das paredes vasculares e dos órgãos internos, aparentemente, deve-se à deposição de componentes da matriz extracelular e ao aumento progressivo do teor de colágeno. Assim, o tecido subcutâneo é parcialmente substituído por colágeno. Essa atividade aumentada dos fibroblastos está relacionada ao estímulo por citocinas inflamatórias, como o TGFbeta (STICHERLING, 2012).

Por se tratar de uma doença rara, a esclerodermia possui tratamento limitado, uma vez que há certa dificuldade em avaliar as melhorias da doença e que não haja parâmetros que sejam universalmente utilizados (FETT, 2013). Como não há tratamento modificador da doença definitivo e que aborde a patogenia básica que a envolve, o foco da terapêutica é tratar as manifestações órgãos específicas (ALMEIDA, 2014).

$\mathrm{Na}$ terapêutica modificadora da doença, tem-se fármacos muito utilizados e que demonstram eficácia em alguns aspectos particulares. Há terapias imunomoduladoras, que podem ser realizadas com Ciclofosfamida, Micofenolato de mofetil, Metotrexato, Azatioprina, entre outros (ALMEIDA, 2014).

A Ciclofosfamida interfere na divisão e na proliferação celular, diminuindo os linfócitos B e T (QUILLINAN; DENTON, 2009). É o agente imunossupressor utilizado há mais tempo e possui vários estudos sobre tratamento da ES (HUNZELMANN et al., 1997). Seu uso é recomendado para tratamento da doença intersticial pulmonar de pacientes portadores de ES (KOWAL-BIELECKA et al., 2009). Dessa forma, deve ser considerada a primeira linha no tratamento de pacientes com ES e que possuam sinais precoces de progressão da doença a nível pulmonar. No caso, descreve-se que a paciente recebeu pulso de Ciclofosfamida, tal medicação também utilizada para tratamento de LES contribui com a alta melhorada que a paciente recebeu.

O micofenolato de mofetil também está relacionado à diminuição de linfócitos $\mathrm{B}$ e $\mathrm{T}$, uma vez que previnem a sua proliferação (TOPAL; DHURAT, 2013). Possui efeitos benéficos nas manifestações cutâneas e diminuição na progressão para manifestações pulmonares da doença (NIHTYANOVA et al., 2007).

Pode-se lançar mão de terapias biológicas, que utilizam anticorpos, e terapias antifibróticas também. Devese avaliar o perfil e as manifestações mais importantes de cada paciente, a fim de instituir a terapêutica mais eficaz e direcionada (ALMEIDA, 2014).

\section{CONSIDERAÇÕES FINAIS}

Tanto o LES como a AS são patologias que isoladamente podem cursar desde forma leve até formas mais graves, ambas doenças auto-imunes que requerem tratamento e acompanhamento a fim de tentar reduzir progressão e complicações. Após a exposição e discussão do caso abordados neste relato, evidencia-se que apesar de ser uma condição clínica rara, a síndrome de sobreposição do Lúpus e Esclerose sistêmica deve ser exposta para conhecimento sobre o caso, pois ainda existem poucas publicações a respeito desse assunto. Nesse sentido é importante, chamar a atenção sobre o tema e incentivar a pensar sobre essa possibilidade diagnóstica em pacientes reumatológicos, bem como suscitar discussões sobre seu tratamento e processo de reabilitação intra-hospitalar e programação de estratégias pós altas. 


\section{REFERÊNCIAS}

1. ABRÃO ALP, SANTANAB CM, BEZERRA ACB, et al. O que o reumatologista deve saber sobre as manifestações orofaciais das doenças reumáticas autoimunes. Revista Brasileira de Reumatologia, v. 56, n. 5, p. 441-450, 2016.

2. ADINOLFI A, VALENTINI E, CALABRESI E, et al. One year in review 2016: systemic lupus erythematosus. ClinExpRheumatol, v. 34, p. 569-574, 2016.

3. ALMEIDA JIS. Esclerodermia: atualização terapêutica. 2014. Dissertação de Mestrado.

4. BALBIR-GURMAN A, BRAUN-MOSCOVICI Y. Scleroderma-new aspects in pathogenesis and treatment. Best Practice \& Research Clinical Rheumatology, v. 26, n. 1, p. 13-24, 2012.

5. BELMONT HM, ABRAMSON SB, LIE JT. Pathology and pathogenesis of vascular injury in systemic lupus erythematosus. Interactions of inflammatory cells and activated endothelium. Arthritis \& Rheumatology, v. 39, n. 1, p. 9-22, 1996.

6. BORTOLUZZI A, SCIRE CA, BOMBARDIERI S, et al. Development and validation of a new algorithm for attribution of neuropsychiatric events in systemic lupus erythematosus. Rheumatology, v. 54, n. 5, p. 891-898, 2014.

7. BORBA EF, LATORRE LC, BRENOL JCT, et al. Consenso de Lúpus Eritematoso Sistêmico. RevBrasReumatol, v. 48, n.4, p. 196-207, 2008.

8. COSTALLAT LTL, COIMBRA AMV. Lúpus eritematoso sistêmico: análise clínica e laboratorial de 272 pacientes em um hospital universitário (1973-1992). RevBrasReumatol, v. 35, n. 1, p. 23-29, 1995.

9. DORIA A, IACCARINO L, LA MONTAGNA G, et al. Clinical profile and direct medical cost of care of adults presenting with systemic lupus erythematosus in Italy. Clinical and experimental rheumatology, v. 33, n. 3, p. 375384, 2015.

10. FASSBINDER T, SAUNDERS U, MICKHOLZ E, et al. Differential effects of cyclophosphamide and mycophenolate mofetil on cellular and serological parameters in patients with systemic lupus erythematosus. Arthritis research \& therapy, v. 17, n. 1, p. 92, 2015.

11. FETT N. Scleroderma: nomenclature, etiology, pathogenesis, prognosis, and treatments: facts and controversies. Clinics in dermatology, v. 31, n. 4, p. 432-437, 2013.

12. Günther $\mathrm{C}$, Meurer $\mathrm{M}$, Stein A, et al. Familial chilblain lupus-a monogenic form of cutaneous lupus erythematosus due to a heterozygous mutation in TREX1. Dermatology, v. 219, n. 2, p. 162-166, 2009.

13. HORIMOTO AMC, MATOS ENN, COSTA MR, et al. Incidência e prevalência de esclerose sistêmica em Campo Grande, Estado de Mato Grosso do Sul, Brasil. Revista Brasileira de Reumatologia, v. 57, n. 2, p. 107-114, 2017.

14. HUI-YUEN JS, REDDY A, TAYLOR J, et al. Safety and efficacy of belimumab to treat systemic lupus erythematosus in academic clinical practices. The Journal of rheumatology, v. 42, n. 12, p. 2288-2295, 2015.

15. HUMMERS LK, HALL A, WIGLEY FM, et al. Abnormalities in the regulators of angiogenesis in patients with scleroderma. The Journal of rheumatology, v. 36, n. 3, p. 576-582, 2009.

16. HUNZELMANN N, ANDERS S, FIERLBECK G, et al. Double-blind, placebo-controlled study of intralesional interferon gamma for the treatment of localized scleroderma. Journal of the American Academy of Dermatology, v. 36, n. 3, p. 433-435, 1997.

17. JÄRVINEN TM, HELLQUIST A, ZUCCHELLI M. et al. Replication of GWAS-identified systemic lupus erythematosus susceptibility genes affirms B-cell receptor pathway signalling and strengthens the role of IRF5 in disease susceptibility in a Northern European population. Rheumatology, v. 51, n. 1, p. 87-92, 2011.

18. KAYSER C, ANDRADE LEC. Esclerose sistêmica. Sato E. Guias de Medicina Ambulatorial e HospitalarReumatologia. São Paulo: Manole, p. 111-20, 2004.

19. KOWAL-BIELECKA O, FRANSEN J, AVOUAC J, et al. EULAR recommendations for the treatment of systemic sclerosis: a report from the EULAR Scleroderma Trials and Research group (EUSTAR). Annals of the rheumatic diseases, v. 68, n. 5, p. 620-628, 2009.

20. KRISHNASWAMY G, KELLEY J, YERRA L, et al. Human endothelium as a source of multifunctional cytokines: molecular regulation and possible role in human disease. Journal of interferon \& cytokine research, v. 19, n. 2, p. 91-104, 1999.

21. JÜNGER M, KAPP A, KAUFMANN R, et al. Cutaneous lupus erythematosus. Part 1: clinical manifestations and classification. Der Hautarzt; Zeitschrift furDermatologie, Venerologie, und verwandteGebiete, v. 57, n. 3, p. 251 67; quiz 268, 2006.

22. LIN HK, WANG JD, FU LS. Juvenile diffuse systemic sclerosis/systemic lupus erythematosus overlap syndrome-a case report. Rheumatology international, v. 32, n. 6, p. 1809-1811, 2012.

23. LORENZONI MD, MARTINS MR. Esclerodermia: diagnóstico e classificação, 2010.

24. MAVRAGANI CP, FRAGOULIS GE, SOMARAKIS G, et al. Clinical and laboratory predictors of distinct histopathogical features of lupus nephritis. Medicine, v. 94, n. 21, 2015.

25. MCMAHAN ZH, HUMMERS LK. Systemic sclerosis-challenges for clinical practice. Nature Reviews Rheumatology, v. 9, n. 2, p. 90-100, 2013.

26. MOREIRA IC, MOREIRA LD, ABADIA LG et al. Lúpus eritematoso sistêmico e sua interferência na homeostasia corporal. Revista Educação em saúde, v. 13, 2017. 
27. NAKASHIMA CAK, GALHARDO APS, FIORENZANO JFM et al. Incidência e aspectos clínico-laboratoriais do lúpus eritematoso sistêmico em cidade do sul do Brasil. Rev. Bras. Reumatol, v. 51, n. 3, p. 235-239, 2011.

28. NIGHTINGALE AL, FARMER RDT, DE VRIES CS. Incidence of clinically diagnosed systemic lupus erythematosus 1992-1998 using the UK General Practice Research Database. Pharmacoepidemiology and drug safety, v. 15, n. 9, p. 656-661, 2006.

29. NIHTYANOVA SI, BROUGH GM, BLACK CM. et al. Mycophenolate mofetil in diffuse cutaneous systemic sclerosis-a retrospective analysis. Rheumatology, v. 46, n. 3, p. 442-445, 2006.

30. NORGAARD JC, STENGAARD-PEDERSEN K, NORGAARD M et al. Antimalarials in the treatment of systemic lupus erythematosus: a registry-based cohort study in Denmark. Lupus, v. 24, n. 3, p. 299-306, 2015.

31. OLIVEIRA FM. Reconhecimento de Padrão em Pacientes com Esclerose Sistêmica por Sistemas Fuzzy. 2017. Tese de Doutorado. Universidade Federal do Rio de Janeiro.

32. PONS-ESTEL GJ, ALARCÓN GS, SCOFIELD L, et al. Understanding the epidemiology and progression of systemic lupus erythematosus. In: Seminars in arthritis and rheumatism. WB Saunders, 2010. p. 257-268.

33. QUILLINAN NP, DENTON CP. Disease-modifying treatment in systemic sclerosis: current status. Current opinion in rheumatology, v. 21, n. 6, p. 636-641, 2009.

34. REDDY V, CAMBRIDGE C, ISENBERG DA, et al. Internalization of rituximab and the efficiency of B cell depletion in rheumatoid arthritis and systemic lupus erythematosus. Arthritis \& rheumatology, v. 67, n. 8, p. 2046-2055, 2015.

35. RUS V. The epidemiology of systemic lupus erythematosus. Dubois' lupuserythematosus, p. 34-44, 2007.

36. SATO EI, NATOUR J, MARTINELI VP, et al. Seguimento clínico e laboratorial de 132 pacientes com lúpus eritematoso sistêmico. Rev. Bras. Reumatol, v. 31, n. 2, p. 57-62, 1991.

37. STICHERLING M. Systemic sclerosis-dermatological aspects. Part 1: Pathogenesis, epidemiology, clinical findings. JDDG: Journal der DeutschenDermatologischen Gesellschaft, v. 10, n. 10, p. 705-716, 2012.

38. TOROK KS. Pediatric scleroderma: systemic or localized forms. Pediatric clinics of North America, v. 59, n. 2, p. 381-405, 2012.

39. TYNDALL A, MATUCCI-CERINIC M, MÜLLER-LADNER U. Future targets in the management of systemic sclerosis. Rheumatology, v. 48, n. suppl_3, p. iii49-iii53, 2009. 